\title{
C-C Motif Chemokine Ligand 15 May Be a Useful Biomarker for Predicting the Prognosis of Patients with Chronic Hypersensitivity Pneumonitis
}

\author{
Masako Watanabe ${ }^{a}$ Yasushi Horimasu $^{a}$ Hiroshi Iwamoto $^{a}$ Kakuhiro Yamaguchi $^{a}$ \\ Shinjiro Sakamoto ${ }^{a}$ Takeshi Masuda ${ }^{a}$ Taku Nakashima ${ }^{a}$ Shintaro Miyamoto ${ }^{a}$ \\ Shinichiro Ohshimo $^{b}$ Kazunori Fujitaka ${ }^{a}$ Hironobu Hamadac Nobuoki Kohno ${ }^{d}$ \\ Noboru Hattori ${ }^{a}$ \\ a Department of Molecular and Internal Medicine, Graduate School of Biomedical and Health Sciences, Hiroshima \\ University, Minami-ku, Hiroshima, Japan; ${ }^{b}$ Department of Emergency and Critical Care Medicine, Graduate School of \\ Biomedical and Health Sciences, Hiroshima University, Minami-ku, Hiroshima, Japan; ' ${ }^{`}$ Department of Physical Analysis \\ and Therapeutic Sciences, Graduate School of Biomedical and Health Sciences, Hiroshima University, Minami-ku, \\ Hiroshima, Japan; ${ }^{d}$ Hiroshima Cosmopolitan University, Minami-ku, Hiroshima, Japan
}

\section{Keywords}

Bronchoalveolar lavage fluid C-C motif chemokine ligand $15 \cdot$ Prognostic biomarker · Enzyme-linked immunosorbent assay

\footnotetext{
Abstract

Background: Chronic hypersensitivity pneumonitis (CHP) is characterized by lymphocytic inflammation and progressive fibrosis of the lung caused by a variety of inhaled antigens. Due to the difficulty of accurately diagnosing CHP, and the poor prognosis associated with the condition, a novel clinical biomarker is urgently needed. Objective: To investigate the usefulness of C-C motif chemokine ligand 15 (CCL15), which had been demonstrated to highly express in the lungs of CHP patients, as a clinical biomarker for CHP. Method: Immunohistochemical investigations were performed on lung tissue from CHP patients, and CCL15 levels in serum and bronchoalveolar lavage fluid (BALF) were measured via the enzyme-linked immunosorbent assay. Results: Immunohis-
}

tochemistry investigations revealed high CCL15 expression in the lungs of CHP patients. Serum CCL15 levels in CHP patients $(29.1 \pm 2.1 \mu \mathrm{g} / \mathrm{mL})$ were significantly higher than those of idiopathic pulmonary fibrosis patients $(19.7 \pm 1.3 \mu \mathrm{g} / \mathrm{mL}$, $p=0.01)$ and healthy subjects $(19.5 \pm 1.7 \mu \mathrm{g} / \mathrm{mL}, p=0.003)$. When BALF CCL15 level was divided by BALF albumin (Alb) level (BALF CCL15/Alb), it was significantly inversely correlated with forced vital capacity $(\beta=-0.47, p=0.0006)$, percentage of predicted carbon monoxide diffusion capacity of the lung ( $\beta=-0.41, p=0.0048$ ), and BALF lymphocyte count $(\beta=-0.34, p=0.01)$ in CHP patients. Multivariate Cox proportional hazards analysis revealed that high BALF CCL15/Alb and poor prognosis were statistically significantly independently correlated in CHP patients (HR 1.1, 95\% Cl 1.03-1.18, $p=0.004)$. Conclusion: The results of the current study suggest that CCL15 may be a useful prognostic biomarker for CHP. CCL15 was highly expressed in the lung tissue of CHP patients, and BALF CCL15/Alb was significantly associated with CHP prognosis.

\section{KARGER}

(c) 2019 S. Karger AG, Basel

E-Mail karger@karger.com

www.karger.com/res
Yasushi Horimasu

Department of Molecular and Internal Medicine

Graduate School of Biomedical and Health Sciences, Hiroshima University

1-2-3 Kasumi, Minami-ku, Hiroshima 734-8551 (Japan)

E-Mail yasushi17@ hiroshima-u.ac.jp 


\section{Introduction}

Chronic hypersensitivity pneumonitis (CHP) is characterized by lymphocytic inflammation and progressive fibrosis predominantly involving the lung interstitium caused by a broad range of inhaled antigens [1-3]. Centrilobular nodules predominantly distributed in the upper lobes accompanied by reticular shadows with or without honeycombing are typical findings in high-resolution computed tomography of CHP patients $[4,5]$.

Despite the features described above, accurate diagnosis of CHP is often difficult for several reasons. The identification of causative inhaled antigens requires careful and detailed interviewing. Second, radiological findings of CHP often mimic those of idiopathic pulmonary fibrosis (IPF) or other fibrosing interstitial pneumonias $[3,6]$. Third, there are no established diagnostic criteria for CHP that are applied worldwide. Nonetheless, accurate and prompt diagnosis is of critical importance, because rapid deterioration of pulmonary function occurs in some patients and is associated with a poor prognosis [711], and therapeutic strategies for CHP differ from those for other fibrosing interstitial pneumonias. Avoidance or minimization of exposure to the causative antigen is the basic principle in the management of CHP [3]. In contrast, anti-inflammatory or antifibrotic agents should be used in patients with other fibrosing interstitial pneumonias. Thus, there is a strong need for a novel clinical biomarker to facilitate accurate diagnosis and predict outcomes of CHP patients.

We recently performed gene expression analysis using surgically resected lung tissue from 9 CHP patients [12]. We identified several upregulated genes in diseased lungs compared to control lungs. Among them, we focused on $\mathrm{C}-\mathrm{C}$ motif chemokine ligand 15 (CCL15), a secreted chemoattractant protein belonging to the macrophage inflammatory protein-1 family [13], because CCL15 mRNA expression was 20-fold higher or greater in the lungs of 6 of the $9 \mathrm{CHP}$ patients than in the lungs of the controls [12]. Interestingly, we also found that the ratio of CCL15 mRNA expression in the lung tissue of IPF patients to that of control lung tissue was $<0.05$ in 6 out of 7 IPF patients in another gene expression analysis [14]. Based on these contrasting mRNA expression profiles, we speculated that the involvement of CCL15 in the pathogenesis of interstitial inflammation and/or fibrosis in CHP may differ from that of IPF. Thus, CCL15 may be a useful biomarker for CHP.

Based on the aforementioned findings and speculations, we designed the present study to investigate the usefulness of CCL15 as a clinical biomarker for CHP.

\section{Materials and Methods}

\section{Subjects}

The subjects enrolled in the study were 51 Japanese patients with CHP, 78 with IPF, and 69 healthy control subjects. All CHP and IPF patients were diagnosed at Hiroshima University Hospital between 2001 and 2014. Diagnoses of CHP and IPF were made in accordance with the diagnostic criteria for CHP proposed by Yoshizawa et al. [1] and the ATS/ERS/JRS/ALAT joint statement for IPF in 2011 [15], respectively. Briefly, CHP is diagnosed when 3 or more of the following conditions (including either [i] or [ii], [iii] or [iv], and [v] or [vi]) are fulfilled: (i) recurrence of symptoms by environmental provocation or antigen inhalation, (ii) presence of antibodies and/or lymphocytic proliferation targeting the specific antigen, (iii) evidence of pulmonary fibrosis with or without granulomas in histopathological analysis, (iv) honeycomb pattern on $\mathrm{CT},(\mathrm{v})$ progressive deterioration of restrictive pulmonary function impairment over 1 year, and (vi) persistence of respiratory symptoms related to the disease for $>6$ months [1]. We also investigated whether the patients with CHP enrolled in this study fulfilled the newer diagnostic criteria proposed by Vasakova et al. [16].

The healthy subjects were recruited from a pool of recipients of health checkups at the hospital consisting of a disease screening questionnaire, chest X-ray, pulmonary function tests, and electrocardiography. Those with apparent lung diseases such as COPD or interstitial lung diseases were excluded. Additionally, those with lung cancer, colon cancer, liver cancer, asthma, or chronic renal failure were also excluded from both patient groups and the control group, because these diseases have been reported to be associated with increased expression of CCL15 [17-24]. The present study was approved by the Ethics Committee of Hiroshima University Hospital (approval number 326) and conducted in accordance with the ethical standards established in the Helsinki Declaration of 1975. All participants provided written informed consent to use their samples for this study and to publish the results.

\section{Serum and Bronchoalveolar Lavage Sampling Procedure}

Serum samples were obtained from blood drawn during initial patient assessments or during health checkups. Freshly acquired blood samples were promptly centrifuged and cryopreserved at $-80^{\circ} \mathrm{C}$ until they were analyzed. Bronchoalveolar lavage (BAL) was performed under local anesthesia, by introducing $50 \mathrm{~mL}$ of saline into the lung and promptly drawing it out again via a bronchoscope. These procedures were repeated 3 times, and $150 \mathrm{~mL}$ of saline in total was introduced during BAL. The BAL target region was either the right middle lobe or the lingular segment of left lung, depending on which was the most severely affected as determined in accordance with the findings of high-resolution computed tomography. The retrieved saline was centrifuged promptly, and the supernatant was cryopreserved at $-80^{\circ} \mathrm{C}$ until it was analyzed.

\section{Pulmonary Function Tests}

Spirometry and carbon monoxide diffusion capacity of the lung (DLco) measurements were performed in accordance with the recommendations of the American Thoracic Society [25]. Annual declines in forced vital capacity (FVC) and DLco were calculated by dividing the slope of the regression lines for FVC or DLco by the baseline values, as previously described [12]. 
Table 1. Baseline patient characteristics

\begin{tabular}{lccc}
\hline & CHP & IPF & Control \\
\hline Sample, $n$ & 51 & 78 & 69 \\
Serum/BALF available, $n$ & $46 / 51$ & $78 / 53$ & $69 / 0$ \\
Gender, male/female, $n$ & $29 / 22$ & $69 / 9$ & $59 / 10$ \\
Age, years & $68.4 \pm 1.2$ & $68.9 \pm 1.0$ & $62.0 \pm 0.9$ \\
Smoking history, yes/no, $n$ & $28 / 23$ & $66 / 12$ & $54 / 15$ \\
Pulmonary function tests & & & $3.5 \pm 0.1$ \\
FVC, L & $2.2 \pm 0.1$ & $2.5 \pm 0.1$ & $98.8 \pm 2.4$ \\
\%FVC, \% & $75 \pm 3.4$ & $74.5 \pm 2.4$ & $\mathrm{NA}$ \\
DLco, mL/min/mm Hg & $10.2 \pm 0.7$ & $10.8 \pm 0.5$ & $\mathrm{~N}$ \\
\%DLco, \% & $47.7 \pm 2.6$ & $46.3 \pm 1.9$ & $\mathrm{NA}$ \\
BALF & & & $\mathrm{NA}$ \\
Total cell, $10^{4} / \mathrm{mL}$ & $31.4 \pm 3.8$ & $21.3 \pm 2.1$ & $\mathrm{NA}$ \\
Lymphocyte, $10^{4} / \mathrm{mL}$ & $9.9 \pm 2.2$ & $2.3 \pm 0.5$ & $0 / 0 / 69$ \\
Lymphocyte, \% & $28.2 \pm 3.2$ & $10.4 \pm 1.2$ & $0 / 0 / 69$ \\
Biopsy, VATS/TBLB/NA, $n$ & $19 / 29 / 3$ & $14 / 33 / 31$ & $0 / 0 / 78$ \\
Antibodies, Avian/T. asahii/NA detective, $n$ & $13 / 8 / 30$ & & \\
\hline
\end{tabular}

Numerical data are represented as mean \pm SEM.

CHP, chronic hypersensitivity pneumonitis; IPF, idiopathic pulmonary fibrosis; BALF, bronchoalveolar lavage fluid; FVC, forced vital capacity; \%FVC, percentage of predicted FVC; DLco, carbon monoxide diffusion capacity of the lung; \%DLco, percentage of predicted DLco; VATS, video-assisted thoracic surgery; TBLB, transbronchial lung biopsy; Avian, Avian-specific antibodies includes IgG or IgA for pigeon, parrot or budgerigar; T. asahii, anti-Trichosporon asahii antibody; NA, not assessed.

Immunohistochemistry Staining for CCL15

Lung tissue sections from CHP patients and IPF were obtained from surgical lung biopsy specimens that had been acquired for diagnostic purposes. Control lung tissue sections were obtained from the healthy areas of lungs that had been surgically resected along with lung tumors for therapeutic purposes. These lung tissue sections were stained using the ENVISION+ Kit/horseradish peroxidase (HRP; Dako, Tokyo, Japan) as previously described [14]. Anti-CCL15 antibody (1:300, Lot ID: 52039; LifeSpan BioSciences Inc., Seattle, WA, USA) was added after blocking of endogenous peroxidase and proteins. The sections were then incubated with HRP-labeled anti-goat immunoglobulin (Ig) G secondary antibody followed by the addition of substrate-chromogen and counterstaining with hematoxylin.

\section{Measurement of CCL15 and Albumin Concentrations}

CCL15 levels in serum and BAL fluid (BALF) were measured using commercially available enzyme-linked immunosorbent assay kits in accordance with the manufacturer's instructions (R\&D Systems, Minneapolis, MN, USA). BALF albumin (Alb) levels were measured using a commercially available immunoturbidity kit (AutoWako Microalbumin; Wako Pure Chemical Industries, Osaka, Japan).

\section{Statistical Analysis}

For numerical variables, means \pm SEM were calculated. Either the Mann-Whitney U test or Pearson's chi-square test was used to assess comparisons between groups, as appropriate. Receiver operating characteristic curve analysis was performed to determine the appropriate CCL15 threshold for predicting 5-year survival.
The difference in survival between the 2 groups was evaluated using the log-rank test. The predictive ability of CCL15 was tested via a Cox proportional hazards regression model. All data analyses were performed with JMP PRO statistical software version 12.2.0 (SAS Institute Inc., Cary, NC, USA).

\section{Results}

\section{Baseline Characteristics}

The baseline characteristics of the 51 patients with CHP, 78 patients with IPF, and 69 healthy subjects included in the current study are shown in Table 1. Among these subjects, serum samples were available for 46 of the 51 patients with CHP, all 78 patients with IPF, and all 69 healthy subjects. BALF samples were available for all 51 patients with CHP, and 53 of the 78 patients with IPF. Compared to the IPF group, the CHP group contained less male subjects, and the subjects had lighter smoking histories and more lymphocytes in BALF.

As shown in online supplementary Table 1 (for all online suppl. material, see www.karger.com/ doi/10.1159/000500576), there were no significant differences in the baseline characteristics of the serum analysis groups and the BALF analysis groups for $\mathrm{CHP}$ or IPF. 


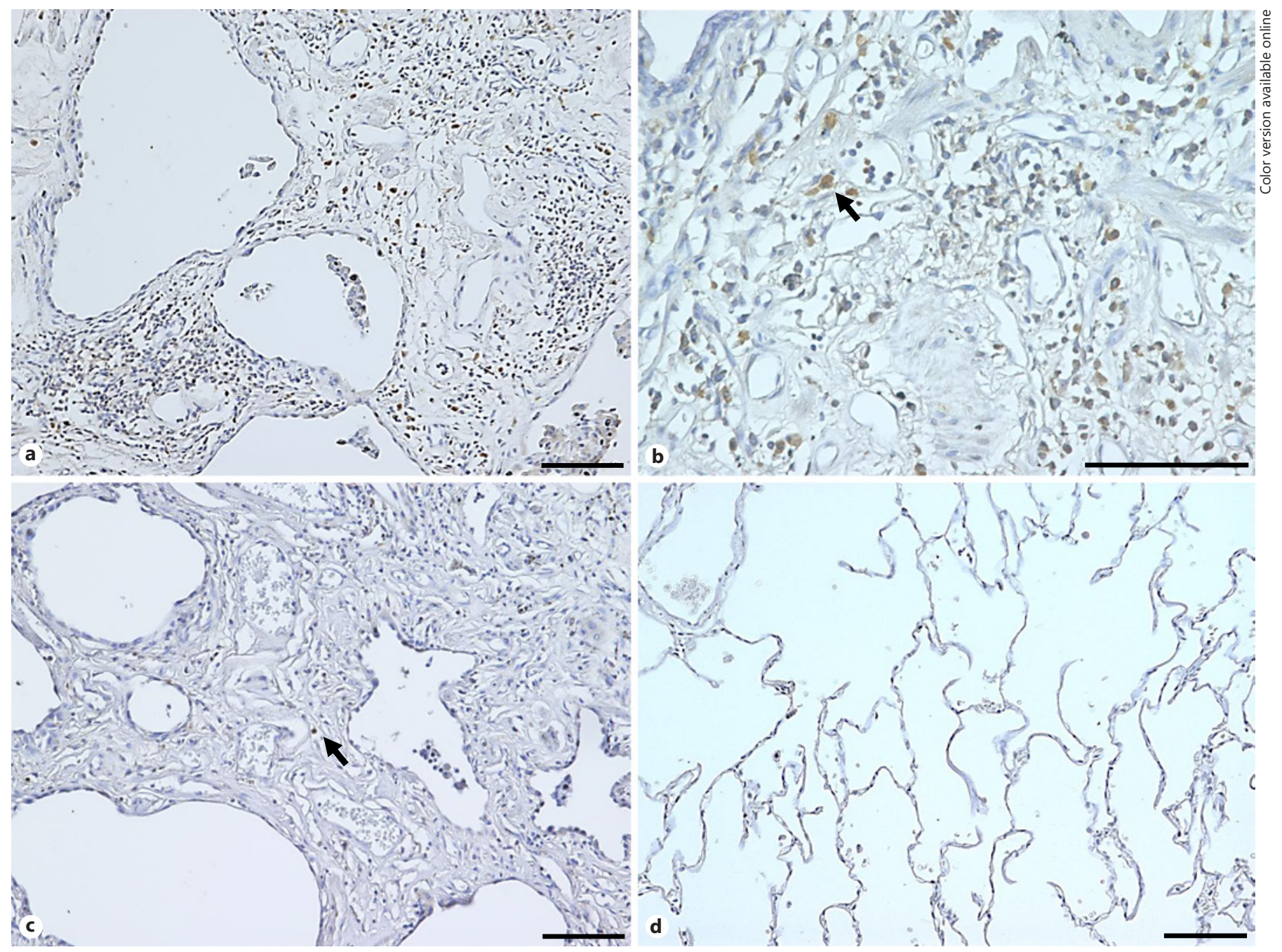

Fig. 1. Immunohistochemistry staining for CCL15 in CHP, IPF, and control lungs. Scale bar $=100 \mu \mathrm{m}$. CCL15positive cells are indicated by black arrows. a CHP. b High-power field in CHP. c IPF. d Control.

\section{Diagnosis of CHP}

As described above, all patients with CHP fulfilled the diagnostic criteria proposed by Yoshizawa et al. [1]. According to Vasakova et al. [16] criteria, 20, 8, and 23 patients exhibited "confident clinically diagnosed HP," "probable HP," and "possible HP," respectively. Seven of the 8 patients with "probable HP" and 22 of the 23 patients with "possible HP" showed pathological findings compatible with HP. Therefore, 49 of the 51 patients with CHP were considered to have "confident clinically diagnosed HP" and/or "definite HP" on the basis of Vasakova's criteria. As shown in Table 1, specific IgG or IgA for pigeon, parrot, budgerigar, and/or Trichosporon asahii was detected in 21 patients with CHP.
CCL15 May Be a Useful Biomarker for Predicting the Prognosis of CHP

\section{CCL15 Expression Is Increased in the Lungs of CHP}

\section{Patients}

Immunohistochemistry results revealed that CCL15 was markedly expressed in plasma cells and macrophages infiltrating the fibrosing interstitium and macrophages in the alveolar space in the lungs of CHP patients (Fig. 1a, b). Week CCL15 expression was detected in the interstitial plasma cells and macrophages in the lungs of IPF patients (Fig. 1c). CCL15 expression was not detected in the control lungs (Fig. 1d).

Serum CCL15 Is Significantly Higher in CHP Patients than in IPF Patients and Healthy Controls

Serum CCL15 in CHP patients $(29.1 \pm 2.1 \mu \mathrm{g} / \mathrm{mL})$ was significantly higher than those in IPF patients $(19.7 \pm 1.3$ 


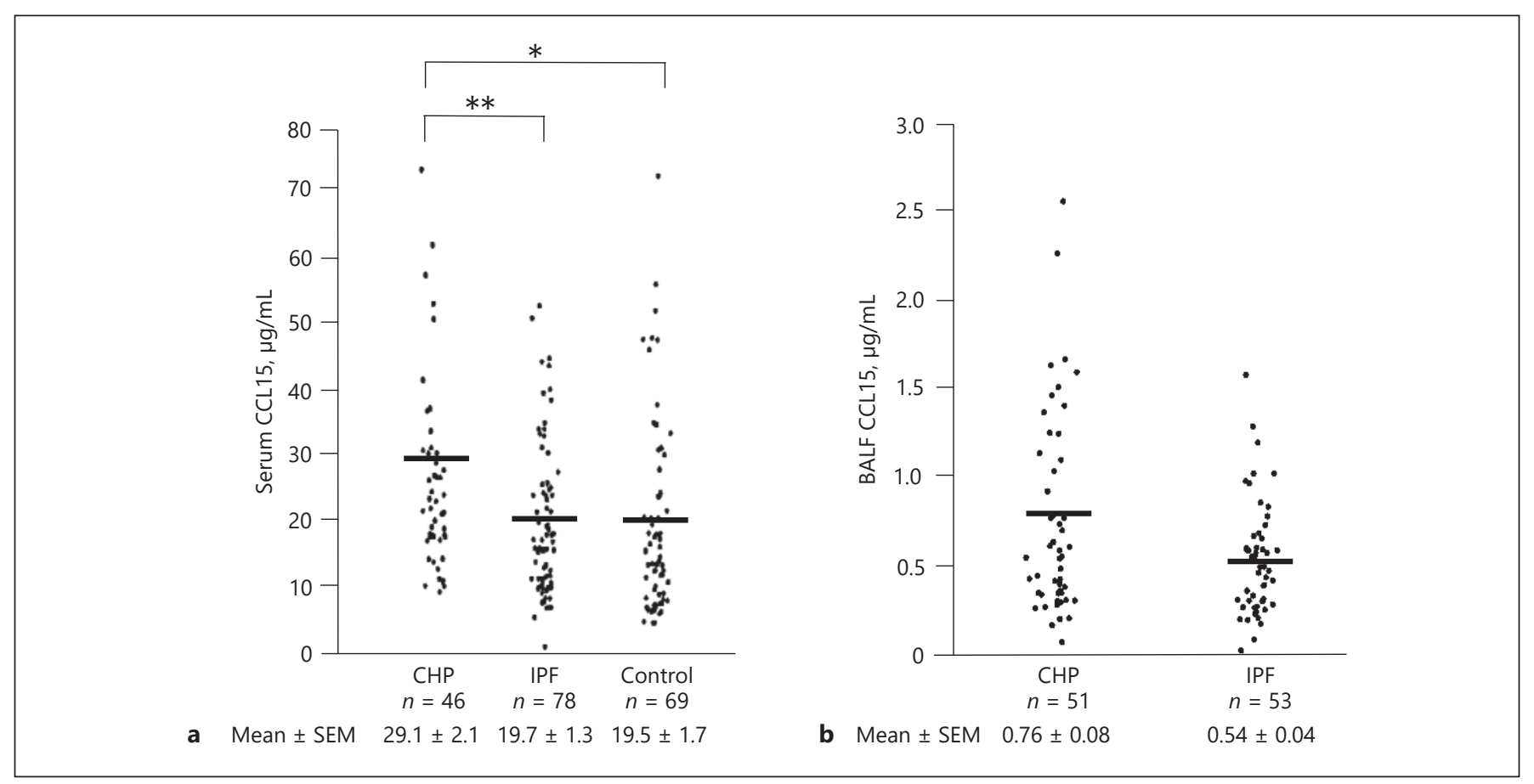

Fig. 2. a In CHP patients, serum CCL15 were significantly higher than they were in IPF patients and healthy subjects. b In CHP patients, BALF CCL15 tended to be higher than they were in IPF patients $\left(p=0.06\right.$, Mann-Whitney U test). ${ }^{*} p=0.003,{ }^{* *} p=0.01$,
Mann-Whitney U test with Bonferroni correction. CCL15, C-C motif chemokine ligand 15; CHP, chronic hypersensitivity pneumonitis; IPF, idiopathic pulmonary fibrosis, BALF, bronchoalveolar lavage fluid. $\mu \mathrm{g} / \mathrm{mL} ; p=0.01)$ and healthy subjects $(19.5 \pm 1.7 \mu \mathrm{g} / \mathrm{mL}$; $p=0.003$; Fig. 2a). In CHP patients, BALF CCL15 tended to be higher than they were in IPF patients, although the difference was not statistically significant (Fig. 2b).

\section{BALF CCL15 Was Correlated with Baseline}

Pulmonary Function and with Serial Change in

Pulmonary Function

To assess the role of CCL15 in the development of clinical manifestations of CHP, we investigated whether serum or BALF CCL15 was significantly correlated with clinical characteristics including pulmonary function and BALF cell properties. As shown in Table 2, neither serum nor BALF CCL15 was significantly correlated with pulmonary function or BALF total cell or lymphocyte count, whereas there was a weak correlation between BALF CCL15 and FVC $(p=0.02)$. Importantly, when divided by BALF Alb level, BALF CCL15 was significantly correlated with FVC $(p<0.001)$, percentage of predicted FVC (\%FVC; $p=0.002)$, DLco $(p=0.002)$, and percentage of predicted carbon monoxide diffusion capacity of the lung (\%DLco; $p=0.005$; Table 2 ). BALF CCL15 was also only correlated with BALF lymphocyte counts and BALF lym- phocyte fractions when it was divided by BALF Alb ( $p=$ 0.01 and $p=0.005$, respectively; Table 2). Higher BALF CCL15/BALF Alb (BALF CCL15/Alb) was significantly correlated with more severe annual decline in FVC in CHP patients ( $p=0.0024$; Fig. 3 ). Conversely, neither serum CCL15 nor BALF CCL15 was significantly correlated with pulmonary function or BALF cell properties in IPF patients (online suppl. Table 2).

\section{BALF CCL15/Alb Can Predict CHP Prognosis}

Based on the above results, we surmised that BALF CCL15/Alb may be a useful biomarker for CHP. This method has previously been utilized to remove the variable of dilution and also to remove the influence of serum protein that has infiltrated into the alveolar space from capillary vessels [26]. On the basis of receiver operating characteristic curve analysis, we determined that the appropriate BALF CCL15/Alb threshold for predicting 5-year survival in patients with $\mathrm{CHP}$ was 10.82. As shown in online supplementary Figure 1, the survival rate for patients with a high BALF CCL15/Alb was significantly lower than that for patients with a low BALF CCL15/Alb $(p=0.002)$. To further investigate the prognostic and pre- 
Table 2. Correlations between CCL15 and baseline characteristics in chronic hypersensitivity pneumonitis patients

\begin{tabular}{|c|c|c|c|c|c|c|c|c|c|}
\hline & $t$ & $\beta$ & $p$ value & $t$ & $\beta$ & $p$ value & $t$ & $\beta$ & $p$ value \\
\hline Age, years & -2.06 & -0.3 & $0.046^{*}$ & 0.35 & 0.05 & 0.73 & 1.04 & 0.15 & 0.3 \\
\hline Smoking history, yes & -0.85 & -0.14 & 0.4 & -0.69 & -0.1 & 0.49 & -0.77 & -0.11 & 0.44 \\
\hline BALF CCL15, $\mu \mathrm{g} / \mathrm{mL}$ & 1.76 & 0.26 & 0.09 & - & - & - & - & - & - \\
\hline$\% \mathrm{FVC}, \%$ & -1 & -0.17 & 0.32 & -1.58 & -0.25 & 0.12 & -3.39 & -0.48 & $0.0016^{*}$ \\
\hline $\mathrm{DLco}, \mathrm{mL} / \mathrm{min} / \mathrm{mm} \mathrm{Hg}$ & -1.61 & -0.26 & 0.12 & -1.58 & -0.24 & 0.12 & -3.38 & -0.47 & $0.0016^{*}$ \\
\hline$\%$ DLco, $\%$ & -1.48 & -0.23 & 0.15 & -0.73 & -0.11 & 0.47 & -2.97 & -0.41 & $0.0048^{*}$ \\
\hline BALF total cell, $10^{4} / \mathrm{mL}$ & -0.51 & -0.08 & 0.61 & -0.61 & -0.09 & 0.54 & -0.98 & -0.14 & 0.33 \\
\hline BALF lymphocyte, $10^{4} / \mathrm{mL}$ & 0.1 & 0.02 & 0.92 & -0.2 & -0.03 & 0.84 & -2.55 & -0.34 & $0.01^{*}$ \\
\hline
\end{tabular}

$* p<0.05$, linear regression analysis.

CCL15, C-C motif chemokine ligand 15; BALF, bronchoalveolar lavage fluid; Alb, albumin; FVC, forced vital capacity; \%FVC, percentage of predicted FVC; DLco, carbon monoxide diffusion capacity of the lung; \%DLco, percentage of predicted DLco.

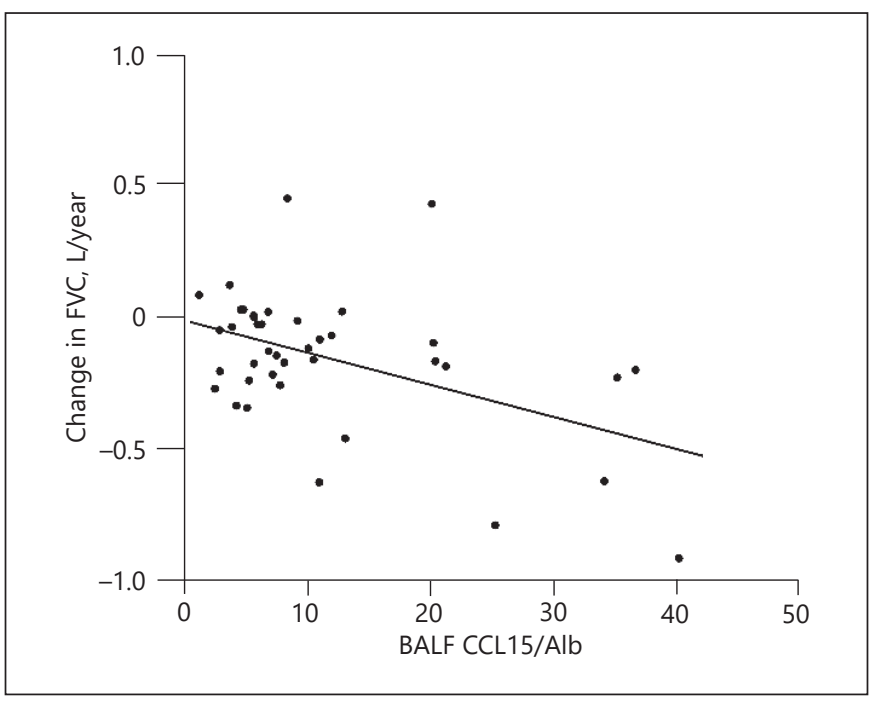

Fig. 3. High BALF CCL15/Alb was significantly associated with change in FVC in CHP patients. $\beta=-0.47, p=0.0024$, linear regression analysis. FVC, forced vital capacity; BALF, bronchoalveolar lavage fluid; CCL15, C-C motif chemokine ligand 15; Alb, albumin.

dictive capacities of BALF CCL15/Alb in CHP patients, we performed Cox proportional hazards analysis. As shown in Table 3, high BALF CCL15/Alb, impaired pulmonary function, and a low lymphocyte count in BALF were significantly correlated with a poor prognosis in CHP patients. However, neither serum nor BALF CCL15 was significantly correlated with prognosis (Table 3 ). Multivariate analysis including BALF CCL15/Alb, FVC, and BALF lymphocyte counts as covariates revealed that the correlation between high BALF CCL15/Alb and poor prognosis was statistically independent in CHP patients ( $p=0.004$; Table 3$)$. BALF CCL15/Alb was not correlated with prognosis in IPF patients (online suppl. Table 3).

\section{Discussion}

The most important finding in the present study was that BALF CCL15/Alb was significantly correlated with prognosis of patients with CHP. However, neither serum nor BALF CCL15 was significantly correlated with prognosis. These results suggest that lung locally derived CCL15 may play an important role in the pathogenesis of CHP. To the best of our knowledge, this is the first report indicating the usefulness of CCL15 as a prognostic biomarker for CHP. In the present study, the expression of CCL15 protein was increased in the lungs of CHP patients. This is consistent with the previously reported increased expression of CCL15 mRNA in the lungs of CHP patients [12].

Higher BALF CCL15/Alb was significantly associated with poorer pulmonary functions at baseline and with a greater decline in pulmonary function over time. As it has been previously reported that the major biological function of CCL15 involves chemotactic activity that attracts 
Table 3. Univariate and multivariate Cox proportional hazards regression model in chronic hypersensitivity pneumonitis patients

\begin{tabular}{|c|c|c|c|c|c|c|}
\hline & \multicolumn{3}{|c|}{ Univariate } & \multicolumn{3}{|c|}{ Multivariate } \\
\hline & HR & $95 \% \mathrm{CI}$ & $p$ value & HR & $95 \% \mathrm{CI}$ & $p$ value \\
\hline Serum CCL15, continuous & 1.00 & $0.95-1.04$ & 0.96 & & & \\
\hline BALF CCL15, continuous & 2.14 & $0.83-4.82$ & 0.11 & & & \\
\hline BALF CCL15/Alb, continuous & 1.12 & $1.06-1.19$ & $0.0001^{*}$ & 1.10 & $1.03-1.18$ & $0.004^{*}$ \\
\hline Gender, male & 0.86 & $0.30-2.63$ & 0.78 & & & \\
\hline Age, years & 1.02 & $0.96-1.09$ & 0.54 & & & \\
\hline Smoking history, yes & 0.56 & $0.19-1.64$ & 0.28 & & & \\
\hline FVC, continuous & 0.42 & $0.17-0.98$ & $0.04^{*}$ & 0.76 & $0.27-2.13$ & 0.59 \\
\hline$\%$ DLco, continuous & 0.96 & $0.92-0.99$ & $0.02^{*}$ & & & \\
\hline BALF total cell, continuous & 1.00 & $0.98-1.02$ & 0.68 & & & \\
\hline BALF lymphocyte, continuous & 0.92 & $0.78-1.00$ & $0.04^{*}$ & 0.96 & $0.82-1.03$ & 0.36 \\
\hline
\end{tabular}

$* p<0.05$, Cox proportional hazards regression model.

CCL15, C-C motif chemokine ligand 15; BALF, bronchoalveolar lavage fluid; Alb, albumin; FVC, forced vital capacity; \%DLco, percentage of predicted carbon monoxide diffusion capacity of the lung.

T-lymphocytes, monocytes, and vascular endothelial cells, mainly via CC chemokine receptor (CCR) 1 or CCR3 $[27,28]$, these pathways may play an important role in the pathogenesis of CHP. CCR1 knockdown has been reported to reduce the secretion of matrix metalloproteinase- 2 or matrix metalloproteinase- 9 by the several types of cancer $[29,30]$. Increased CCR1 expression by neutrophils, monocytes, eosinophils, dendritic cells, activated $\mathrm{T}$ cells, and B lymphocytes is also known to be associated with autoimmune diseases such as multiple sclerosis and rheumatoid arthritis $[13,30]$. These findings suggest that CCL15 may promote inflammatory change in the lungs of CHP patients via interaction with CCR1. These speculations correspond with the results of a previous study involving pathway analysis in CHP, in which we demonstrated that several pathways related to inflammatory responses and immunological diseases were differentially expressed in patients with rapidly progressive CHP [12].

CCR3 is known to be expressed on eosinophils, basophils, mast cells, and Th2 cells [30-32]. Interestingly, other Th2-related chemokines including CCL17 and CCL18 have been reported to be involved in the progression of fibrosis [33-35]. Therefore, we can speculate that CCL15 plays some role in lung fibrosis through its interaction with CCR3.

CCL15 was only significantly correlated with baseline pulmonary function, functional deterioration, and prognosis after it was divided by BALF Alb. CCL15 is not a lung-specific protein, but it is widely expressed in various organs [36-38]. Thus, BALF CCL15 may not only reflect locally-derived CCL15, but also systemically produced CCL15 that is present due to capillary-alveolar permeability. In addition, BALF protein levels measured by enzyme-linked immunosorbent assay can be influenced by the amount of injected saline as well as the amount of recovered saline [39]. In order to negate the influence of systemically produced CCL15 infiltrating the alveolar space and also to remove the variable of dilution inherently associated with the BAL procedure, we divided BALF CCL15 concentration by BALF Alb concentration $[26,40-42]$. As BALF CCL15/Alb is considered to reflect locally derived lung CCL15, these results suggest that locally derived lung CCL15, not systemically-produced CCL15, has an important role in the pathogenesis of CHP and that it can be a useful prognostic biomarker in CHP.

Serum CCL15 were significantly higher in CHP patients than in IPF patients. These results are concordant with the differential expression of CCL15 mRNA in the lungs of CHP patients and IPF patients. It may be that CCL15 is inherently involved in the pathogenesis of CHP, whereas its involvement in the pathogenesis of IPF is limited. Notably, the prognostic predictive capacity of BALF CCL15 was significant in CHP patients but not in IPF patients.

The current study had several limitations. The sample size was limited because the study was conducted at a single facility. The study design was also retrospective, and there was no validation cohort. Thus, multicenter prospective studies are needed to confirm the usefulness of 
CCL15 as a prognostic biomarker in CHP. Despite these limitations, we believe the present study attests to the prognostic predictive capacity of CCL15 in CHP patients.

\section{Conclusion}

The results of the present study suggest that CCL15 may be a useful prognostic biomarker in CHP.

\section{Acknowledgment}

The authors thank Ms. Yukari Iyanaga from Department of Molecular and Internal Medicine Graduate School of Biomedical and Health Science, Hiroshima University for her help. We would like to thank Editage (www.editage.jp) for English language editing.

\section{Statement of Ethics}

The manuscript has not been published or presented elsewhere in part or in entirety, and it is not under consideration by another journal. All study participants provided informed consent, and the study design was approved by the appropriate ethics review board.
We have read and understood your journal's policies, and we believe that neither the manuscript nor the study violates any of these.

\section{Disclosure Statement}

The authors have no conflicts of interest to declare.

\section{Funding Sources}

This research was supported by Japan Society for the Promotion of Science KAKENHI Grant (No. JP 25253057).

\section{Author Contributions}

M.W.: drafted and finalized the manuscript, performed part of the serum measurement, immunohistochemistry staining, and statistical analyses. Y.H., H.I., H.H., N.K., and N.H.: conceived the study and participated in its design and coordination and helped to draft and finalize the manuscript. K.Y.: performed part of the serum measurement and statistical analysis. S.S., T.M., T.N., S.M., S.O., and K.F.: recruited the study subjects, ascertained diagnosis, and helped to draft and finalize the manuscript. All authors read and approved the final manuscript.

\section{References}

1 Yoshizawa Y, Ohtani Y, Hayakawa H, Sato A, Suga M, Ando M. Chronic hypersensitivity pneumonitis in Japan: a nationwide epidemiologic survey. J Allergy Clin Immunol. 1999 Feb;103(2 Pt 1):315-20.

2 Churg A, Muller NL, Flint J, Wright JL. Chronic hypersensitivity pneumonitis. Am J Surg Pathol. 2006 Feb;30(2):201-8.

3 Selman M, Pardo A, King TE Jr. Hypersensitivity pneumonitis: insights in diagnosis and pathobiology. Am J Respir Crit Care Med. 2012 Aug;186(4):314-24.

4 Tateishi T, Ohtani Y, Takemura T, Akashi T, Miyazaki Y, Inase N, et al. Serial high-resolution computed tomography findings of acute and chronic hypersensitivity pneumonitis induced by avian antigen. J Comput Assist Tomogr. 2011 Mar-Apr;35(2):272-9.

5 Silva CI, Müller NL, Lynch DA, Curran-Everett D, Brown KK, Lee KS, et al. Chronic hypersensitivity pneumonitis: differentiation from idiopathic pulmonary fibrosis and nonspecific interstitial pneumonia by using thin-section CT. Radiology. 2008 Jan;246(1):288-97.

6 Churg A, Bilawich A, Wright JL. Pathology of chronic hypersensitivity pneumonitis What is it? What are the diagnostic criteria? Why do we care? Arch Pathol Lab Med. 2018 Jan; 142(1):109-19.
7 Pérez-Padilla R, Salas J, Chapela R, Sánchez M, Carrillo G, Pérez R, et al. Mortality in Mexican patients with chronic pigeon breeder's lung compared with those with usual interstitial pneumonia. Am Rev Respir Dis. 1993 Jul; 148(1):49-53.

8 Vourlekis JS, Schwarz MI, Cherniack RM, Curran-Everett D, Cool CD, Tuder RM, et al. The effect of pulmonary fibrosis on survival in patients with hypersensitivity pneumonitis. Am J Med. 2004 May;116(10):662-8.

9 Miyazaki Y, Tateishi T, Akashi T, Ohtani Y, Inase N, Yoshizawa Y. Clinical predictors and histologic appearance of acute exacerbations in chronic hypersensitivity pneumonitis. Chest. 2008 Dec;134(6):1265-70.

10 Churg A, Sin DD, Everett D, Brown K, Cool C. Pathologic patterns and survival in chronic hypersensitivity pneumonitis. Am J Surg Pathol. 2009 Dec;33(12):1765-70.

11 Ojanguren I, Morell F, Ramón MA, Villar A, Romero C, Cruz MJ, et al. Long-term outcomes in chronic hypersensitivity pneumonitis. Allergy. 2019 May;74(5):944-952.

12 Horimasu Y, Ishikawa N, Iwamoto $\mathrm{H}$, Ohshimo S, Hamada H, Hattori N, et al. Clinical and molecular features of rapidly progressive chronichypersensitivity pneumonitis. Sarcoidosis Vasc Diffuse Lung Dis. 2017;34(1):48-57.
13 Maurer M, von Stebut E. Macrophage inflammatory protein-1. Int J Biochem Cell Biol. 2004 Oct;36(10):1882-6.

14 Horimasu Y, Ishikawa N, Taniwaki M, Yamaguchi $\mathrm{K}$, Hamai K, Iwamoto $\mathrm{H}$, et al. Gene expression profiling of idiopathic interstitial pneumonias (IIPs): identification of potential diagnostic markers and therapeutic targets. BMC Med Genet. 2017 Aug;18(1):88.

15 Raghu G, Collard HR, Egan JJ, Martinez FJ, Behr J, Brown KK, et al.; ATS/ERS/JRS/ALAT Committee on Idiopathic Pulmonary Fibrosis. An official ATS/ERS/JRS/ALAT statement: idiopathic pulmonary fibrosis: evidence-based guidelines for diagnosis and management. Am J Respir Crit Care Med. 2011 Mar;183(6):788-824.

16 Vasakova M, Morell F, Walsh S, Leslie K, Raghu G. Hypersensitivity pneumonitis: perspectives in diagnosis and management. Am J Respir Crit Care Med. 2017 Sep;196(6):6809.

17 Haringman JJ, Smeets TJ, Reinders-Blankert P, Tak PP. Chemokine and chemokine receptor expression in paired peripheral blood mononuclear cells and synovial tissue of patients with rheumatoid arthritis, osteoarthritis, and reactive arthritis. Ann Rheum Dis. 2006 Mar;65(3):294-300. 
18 Joubert P, Lajoie-Kadoch S, Wellemans V, Létuvé S, Tulic MK, Halayko AJ, et al. Expression and regulation of CCL15 by human airway smooth muscle cells. Clin Exp Allergy. 2012 Jan;42(1):85-94.

19 Arakelyan A, Kriegova E, Kubistova Z, Mrazek F, Kverka M, du Bois RM, et al. Protein levels of CC chemokine ligand (CCL)15, CCL16 and macrophage stimulating protein in patients with sarcoidosis. Clin Exp Immunol. 2009 Mar;155(3):457-65.

20 Bodelon C, Polley MY, Kemp TJ, Pesatori AC, McShane LM, Caporaso NE, et al. Circulating levels of immune and inflammatory markers and long versus short survival in early-stage lung cancer. Ann Oncol. 2013 Aug;24(8): 2073-9.

21 Shimizu Y, Dobashi K. CC-chemokine CCL15 expression and possible implications for the pathogenesis of IgE-related severe asthma. Mediators Inflamm. 2012;2012: 475253.

22 Inamoto S, Itatani Y, Yamamoto T, Minamiguchi $\mathrm{S}$, Hirai $\mathrm{H}$, Iwamoto $\mathrm{M}$, et al. Loss of SMAD4 promotes colorectal cancer progression by accumulation of myeloid-derived suppressor cells through the CCL15-CCR1 chemokine axis. Clin Cancer Res. 2016 Jan; 22(2):492-501.

23 Gao Y, Zhou Z, Lu S, Huang X, Zhang C, Jiang $\mathrm{R}$, et al. Chemokine CCL15 mediates migration of human bone marrow-derived mesenchymal stem cells toward hepatocellular carcinoma. Stem Cells. 2016 Apr;34(4):1112-22.

24 Richter R, Forssmann U, Henschler R, Escher S, Frimpong-Boateng A, Forssmann WG. Increase of expression and activation of chemokine CCL15 in chronic renal failure. Biochem Biophys Res Commun. 2006 Jul;345(4):150412.

25 Standardization of Spirometry, 1994 Update. American Thoracic Society. Am J Respir Crit Care Med. 1995 Sep;152(3):1107-36.

26 Tsao TC, Hong J, Huang C, Yang P, Liao SK, Chang KS. Increased TNF-alpha, IL-1 beta and IL-6 levels in the bronchoalveolar lavage fluid with the upregulation of their mRNA in macrophages lavaged from patients with active pulmonary tuberculosis. Tuber Lung Dis. 1999; 79(5):279-85.

27 Berahovich RD, Miao Z, Wang Y, Premack B, Howard MC, Schall TJ. Proteolytic activation of alternative CCR1 ligands in inflammation. J Immunol. 2005 Jun;174(11):7341-51.

28 Hwang J, Kim CW, Son KN, Han KY, Lee KH, Kleinman HK, et al. Angiogenic activity of human CC chemokine CCL15 in vitro and in vivo. FEBS Lett. 2004 Jul;570(1-3):47-51.

29 Yang X, Lu P, Fujii C, Nakamoto Y, Gao JL, Kaneko S, et al. Essential contribution of a chemokine, CCL3, and its receptor, CCR1, to hepatocellular carcinoma progression. Int $\mathrm{J}$ Cancer. 2006 Apr;118(8):1869-76.

30 Wang CL, Sun BS, Tang Y, Zhuang HQ, Cao WZ. CCR1 knockdown suppresses human non-small cell lung cancer cell invasion. J Cancer Res Clin Oncol. 2009 May;135(5): 695-701.

31 Elsner J, Escher SE, Forssmann U. Chemokine receptor antagonists: a novel therapeutic approach in allergic diseases. Allergy. 2004 Dec;59(12): 1243-58

32 Mantovani A, Bonecchi R, Locati M. Tuning inflammation and immunity by chemokine sequestration: decoys and more. Nat Rev Immunol. 2006 Dec;6(12):907-18.

33 Miyazaki Y, Unoura K, Tateishi T, Akashi T, Takemura T, Tomita M, et al. Higher serum CCL17 may be a promising predictor of acute exacerbations in chronic hypersensitivity pneumonitis. Respir Res. 2013 May; 14(1):57

34 Belperio JA, Dy M, Murray L, Burdick MD, Xue YY, Strieter RM, et al. The role of the Th2 CC chemokine ligand CCL17 in pulmonary fibrosis. J Immunol. 2004 Oct;173(7): 4692-8.

35 Prasse A, Probst C, Bargagli E, Zissel G, Toews GB, Flaherty KR, et al. Serum CC-chemokine ligand 18 concentration predicts out- come in idiopathic pulmonary fibrosis. Am J Respir Crit Care Med. 2009 Apr;179(8):71723.

36 Youn BS, Zhang S, Broxmeyer HE, Antol K, Fraser MJ Jr, Hangoc G, et al. Isolation and characterization of LMC, a novel lymphocyte and monocyte chemoattractant human CC chemokine, with myelosuppressive activity. Biochem Biophys Res Commun. 1998 Jun; 247(2):217-22.

37 Youn BS, Zhang SM, Broxmeyer HE, Cooper $\mathrm{S}$, Antol K, Fraser M Jr, et al. Characterization of CKbeta8 and CKbeta8-1: two alternatively spliced forms of human beta-chemokine, chemoattractants for neutrophils, monocytes, and lymphocytes, and potent agonists at CC chemokine receptor 1. Blood. 1998 May; 91(9):3118-26.

38 Pardigol A, Forssmann U, Zucht HD, Loetscher P, Schulz-Knappe P, Baggiolini M, et al. HCC-2, a human chemokine: gene structure, expression pattern, and biological activity. Proc Natl Acad Sci USA. 1998 May; 95(11):6308-13.

39 Rennard SI, Basset G, Lecossier D, O'Donnell KM, Pinkston P, Martin PG, et al. Estimation of volume of epithelial lining fluid recovered by lavage using urea as marker of dilution. J Appl Physiol (1985). 1986 Feb;60(2):532-8.

40 Bargagli E, Penza F, Bianchi N, Olivieri C, Bennett D, Prasse A, et al. Controversial role of RAGE in the pathogenesis of idiopathic pulmonary fibrosis. Respir Physiol Neurobiol. 2009 Feb;165(2-3):119-20.

41 Bratke K, Wendt A, Garbe K, Kuepper M, Julius $\mathrm{P}$, Lommatzsch $\mathrm{M}$, et al. Vitamin D binding protein and vitamin $\mathrm{D}$ in human allergeninduced endobronchial inflammation. Clin Exp Immunol. 2014 Jul;177(1):366-72.

42 Papp Á, Bene Z, Gáspár I, Nagy B Jr, Kádár L Márialigeti T, et al. Decreased VEGF level is associated with elevated ferritin concentration in bronchoalveolar lavage fluid of children with interstitial lung diseases. Respiration. 2015;90(6):443-50 